\title{
GEOMETRIC PROPERTIES OF 2-DIMENSIONAL BROWNIAN PATHS
}

\author{
KRZYSZTOF BuRDZY \\ University of Washington
}

\begin{abstract}
Let $A$ be the set of all points of the plane $\mathbb{C}$, visited by 2 -dimensional Brownian motion before time 1 . With probability 1 , all points of $A$ are "twist points" except a set of harmonic measure zero. "Twist points" may be continuously approached in $\mathbb{C} \backslash A$ only along a special spiral. Although negligible in the sense of harmonic measure, various classes of "cone points" are dense in $A$, with probability 1. "Cone points" may be approached in $\mathbb{C} \backslash A$ within suitable wedges.
\end{abstract}

1. Introduction. It is well known that, with probability 1, the 2-dimensional Brownian trajectory (i.e. the random function $t \rightarrow X(t)$ ) is not differentiable even at a single point. There is no doubt that every initial segment of the Brownian trace (i.e. the set $X([0, t]))$ must be highly irregular. Mandelbrot (1982) confirms this intuition with computer simulations. In his book, the "irregularity" of fractals is measured by their Hausdorff dimension. In this article, a different approach will be taken; it will be shown that the Brownian trace has strange geometric properties.

Itô and McKean (1974), Knight (1981) and Williams (1979) are rich sources of information about Brownian path properties and especially their local properties. The present research has been inspired by some recent activity of Cranston, Hsu and March (1987), El Bachir (1983), Evans (1985), Le Gall (1986, 1987), Mountford (1987) and Shimura $(1984,1985)$. The author himself has published some related results (Burdzy (1985, 1986a,b, 1987 a-f, 1988), Burdzy and Williams (1986)).

The main results are given in Section 2. Their proofs appear in Sections 4-7. They are not arranged in the order of logical implication but it is easy to check that there are no circular arguments in the paper. Section 3, "Preliminaries", should not be totally ignored. It contains many little facts which are not completely trivial.

The main tools used in the paper are two versions of the excursion theory of Markov processes, due to Itô (1972) and Maisonneuve (1975), potential theory and complex analysis. The proofs make use of several difficult and quite recent results of McMillan (1969), Dahlberg (1977), Varadhan and Williams (1985) and an idea of El Bachir (1983) and Le Gall (1986).

The author would like to express his gratitude to Jim Pitman for sending his personal notes taken at a conference. They provided a decisive impulse to write this article. Special thanks go to the Referees for many helpful comments.

Abbreviated title: Brownian paths. Research supported in part by NSF Grant DMS 8419377. 
2. Main results. Let $X$ be the standard complex (=2-dimensional) Brownian motion starting from 0 w.p.1. Denote

$$
\begin{gathered}
\mathbb{R}_{+}=\{x \in \mathbb{R}: x>0\}=\{x \in \mathbb{C}: \Im x=0, \Re x>0\}, \\
A_{t}=X([0, t])=\left\{x \in \mathbb{C}: \exists s \in[0, t] \text { such that } X_{s}=x\right\} .
\end{gathered}
$$

$F_{t}$ will denote the unbounded connected component of $\mathbb{C} \backslash A_{t}$.

$$
G(x, a, b, \epsilon) \stackrel{\mathrm{df}}{=}\{y \in \mathbb{C}: 0<|x-y|<\epsilon, \arg (y-x) \in[a, b]\}
$$

for $x \in \mathbb{C}, a, b \in \mathbb{R}, a \leq b, \epsilon>0$. Let $B_{t}^{F}(a, b)$ be the set of all points $x \in \partial F_{t}$ such that

i) there exists $\epsilon>0$ such that $G(x, a, b, \epsilon) \subset F_{t}$ and

ii) if $a_{1} \leq a, b_{1} \geq b, b_{1}-a_{1}>b-a, \epsilon_{1}>0$ then $G\left(x, a_{1}, b_{1}, \epsilon_{1}\right) \cap A_{t} \neq \emptyset$.

A class $B_{t}(a, b)$ is defined in the same way as $B_{t}^{F}(a, b)$ with the exception that it is required that $x \in A_{t}$ and $G(x, a, b, \epsilon) \subset \mathbb{C} \backslash A_{t}$ rather than $x \in \partial F_{t}$ and $G(x, a, b, \epsilon) \subset F_{t}$.

$$
\begin{gathered}
B_{t}^{F}(c) \stackrel{\mathrm{df}}{=} \bigcup_{\substack{a, b \in \mathbb{R} \\
b-a=c}} B_{t}^{F}(a, b), \\
B_{t}(c) \stackrel{\mathrm{df}}{=} \bigcup_{\substack{a, b \in \mathbb{R} \\
b-a=c}} B_{t}(a, b) .
\end{gathered}
$$

The symbol $B_{t}^{F}(\infty)$ will stand for the set of all twist points of $\partial F_{t}$ i.e., $x \in B_{t}^{F}(\infty)$ if and only if $x \in \partial F_{t}$ and there exists a continuous path $\Gamma=\{\Gamma(s), s \in(0,1)\} \subset F_{t}$ such that $\lim _{s \rightarrow 0} \Gamma(s)=x$ and

$$
-\liminf _{s \rightarrow 0} \arg (\Gamma(s)-x)=\limsup _{s \rightarrow 0} \arg (\Gamma(s)-x)=\infty
$$

Here $\arg$ is chosen so that $\arg (\Gamma(s)-x)$ is a continuous function of $s$. For a function $h: \mathbb{R} \rightarrow \mathbb{R}$ denote

$$
\begin{aligned}
C^{+}(h, \epsilon) & =\{x \in \mathbb{C}:|x|<\epsilon, \Re x>0,0<\Im x<h(\Re x)\}, \\
C^{-}(h, \epsilon) & =\{x \in \mathbb{C}:|x|<\epsilon, \Re x>0,-h(\Re x)<\Im x<0\}, \\
C(h, \epsilon) & =C^{+}(h, \epsilon) \cup C^{-}(h, \epsilon) .
\end{aligned}
$$

Let $V_{t}$ denote the left endpoint of the unbounded component of $\mathbb{R}_{+} \cap F_{t}$. The set of all points in $A_{t}$ at the minimal distance from 1 will be denoted $M_{t}$. It is easy to see that w.p.1, for all $t>0$, the set $A_{t}$ is closed and bounded, $F_{t}$ and $V_{t}$ are well defined, $F_{t}$ is simply connected, and $1 \notin M_{t}$. For every $t>0$, w.p.1, the set $M_{t}$ contains only one point.

Theorem 2.1. i) For every $t>0$, w.p.1, $V_{t} \in B_{t}^{F}(0,0)$.

ii) For $a<0, b>0, b-a<\pi$, w.p. 1 there exists $t>0$ such that $V_{t} \in B_{t}^{F}(a, b)$.

iii) For $a<0, b>0, b-a=\pi$, w.p.1 there is no $t>0$ such that $V_{t} \in B_{t}^{F}(a, b)$.

iv) W.p.1, there are no $a<0, b>0, b-a>\pi, t>0$ such that $V_{t} \in B_{t}^{F}(a, b)$. 
Theorem 2.2. Suppose that $h: \mathbb{R} \rightarrow \mathbb{R}, h(0)=0, h \geq 0$, and $h$ is Lipschitz or nondecreasing. Fix some $t>0$.

i) If $\int_{0}^{1} h(r) r^{-2} d r<\infty$ then w.p.1 there exists $\epsilon>0$ such that $V_{t}+C(h, \epsilon) \subset F_{t}$.

ii) If $\int_{0}^{1} h(r) r^{-2} d r=\infty$ then w.p.1 there is no $\epsilon>0$ such that $V_{t}+C^{+}(h . \epsilon) \subset F_{t}$.

Theorem 2.3. i) For every $t>0$, w.p.1, $M_{t} \subset B_{t}(\pi)$.

ii) For $a, b \in \mathbb{R}, b-a \in(\pi, 3 \pi / 2)$, w.p.1 there exists $t>0$ such that $M_{t}=\left\{X_{t}\right\} \subset$ $B_{t}(a, b)$.

Theorem 2.4. i) For all $a, b \in \mathbb{R}, b-a \in[0, \pi]$, w.p.1, for every $t>0$ the set $B_{t}^{F}(a, b)$ is dense in $\partial F_{t}$.

ii) (Evans (1985)) For every $t>0$, w.p.1, for all $a, b \in \mathbb{R}, b-a>\pi$, one has $B_{t}(a, b)=\emptyset$.

Theorem 2.5. i) (Le Gall (1986, 1987)) For $a, b \in \mathbb{R}, b-a<3 \pi / 2$, w.p. 1 there exists $t>0$ such that $X_{t} \in B_{t}^{F}(a, b)$.

ii) (Burdzy(1985); Shimura(1985)) W.p.1, for all $a, b \in \mathbb{R}, b-a>3 \pi / 2, t>0$, one has $B_{t}(a, b)=\emptyset$.

Theorem 2.6. W.p.1, for every $t>0$,

i) $\partial F_{t} \backslash B_{t}^{F}(\infty)$ has null harmonic measure in $F_{t}$ and, therefore,

ii) $B_{t}^{F}(\infty)$ is dense in $\partial F_{t}$.

3. Preliminaries. This section offers a brief review of some basic notation, definitions, and a few theorems. For more information, consult Blumenthal and Getoor (1968) (Markov processes), Burdzy (1987c) (excursion laws), Doob (1984) (potential theory, Brownian motion, $h$-processes), Itô (1972) (Poisson point processes), Maisonneuve (1975) (exit systems), Naim (1957) (Martin boundary, minimal fine topology), Pommerenke (1975) (complex analysis, prime ends), Williams (1979) (Markov processes, Brownian motion).

i) General notation and definitions. The sets of all integers, real and complex numbers will be denoted $\mathbb{Z}, \mathbb{R}$ and $\mathbb{C}$, resp. By convention, $\mathbb{R}^{2}=\mathbb{C}, \mathbb{R}=\{x \in \mathbb{C}$ : $\Im x=0\}$. Denote $\mathbb{R}_{+}=\{x \in \mathbb{R}: x>0\} \subset \mathbb{C}, D_{*}=\{x \in \mathbb{C}: \Re x>0\}$. The (Euclidean) boundary and the complement (in $\mathbb{C}$ ) of a set $A \subset \mathbb{C}$ will be denoted $\partial A$ and $A^{c} .|\cdot|$ will stand for the Euclidean norm in $\mathbb{C}$ (and $\mathbb{R}$ ).

A function $f$ will be called Lipschitz with constant $\lambda$ if $|f(x)-f(y)|<\lambda|x-y|$ for all $x, y$ in the domain of $f$.

The notation ${ }^{Y} B_{t}^{F}(a, b),{ }^{Y} V_{t}$ etc. will be used instead of $B_{t}^{F}(a, b), V_{t}$ etc. whenever these objects are defined relative to a process $Y$, rather than $X$.

ii) Potential theory and complex analysis. The Green function of a Greenian set $D \subset \mathbb{C}$ will be denoted $G_{D}(x, y)$. For such a domain $D \subset \mathbb{C}$, fix a reference point $z_{0} \in D$ and let $K(x, y)=K_{z_{0}}^{D}(x, y) \stackrel{\text { df }}{=} G_{D}(x, y) / G_{D}\left(x, z_{0}\right)$ for $x, y \in D, x \neq z_{0}$. There exists a unique up to a homeomorphism compactification $D^{M}$ of $D$ such that $K$ may be extended continuously to $\left(D^{M} \backslash\left\{z_{0}\right\}\right) \times D$ and $K(x, \cdot) \equiv K(y, \cdot)$ only if $x=y$. The set $\partial^{M} D \stackrel{\text { df }}{=} D^{M} \backslash D$ will be called the Martin boundary of $D$ and the topology of $D^{M}$ will be called the Martin topology. A point $x \in \partial^{M} D$ will be called minimal if every positive harmonic function in $D$ majorized by $K(x, \cdot)$ is a constant multiple of $K(x, \cdot)$. The set of all minimal Martin boundary points will be denoted $\partial_{1}^{M} D$ and called the minimal Martin boundary. A set $A \subset D$ will be 
called minimal thin in $D$ at $x \in \partial_{1}^{M} D$ if $x$ is an isolated point in the subset $\{x\} \cup A$ of $D^{M}$ or there exists a measure $\nu$ such that

$$
\int_{D} K(x, y) \nu(d y)<\liminf _{\substack{z \rightarrow x \\ z \neq x \\ z \in A}} \int_{D} K(z, y) \nu(d y) .
$$

This definition of minimal thinness is taken from Naim (1957). The minimal fine topology is defined by declaring that $x \in \partial_{1}^{M} D$ is a minimal fine limit point of $A$ if $A$ is not minimal thin in $D$ at $x$.

The Euclidean boundary may be identified with a ("finite") part of the minimal Martin boundary in Lipschitz domains. In simply connected plane domains, the Martin boundary and the minimal Martin boundary coincide and may be identified with the Carathéodory prime end boundary. The Green function is conformal invariant so the Martin kernel $K$, Martin boundary, minimal thin sets, harmonic measure, etc. are conformal invariant as well.

It will be convenient to look at subsets of $\mathbb{C}$ as subsets of the Riemann sphere i.e., the usual one point compactification of $\mathbb{C}$. Thus, for example, both sets $\{x \in$ $\mathbb{C}:|x|<1\}$ and $\{x \in \mathbb{C}:|x|>1\}$ are simply connected and there exists a conformal one-to-one mapping of the first one onto the other. If the complement of an open simply connected set $D \subset \mathbb{C}$ contains at least two distinct points then $D$ is a Greenian domain.

Now, a few geometric criteria for the minimal thin sets will be given.

(1) Suppose that $A \subset D_{*}$ is an open and simply connected set. It is a minimal fine neighborhood of $0 \in \partial_{1}^{M} D_{*}$ if and only if there exists a Lipschitz function $h: \mathbb{R} \rightarrow \mathbb{R}$ such that $h(r)>0$ for $r \neq 0$,

$$
\int_{-1}^{1} h(r) r^{-2} d r<\infty
$$

and for some $\epsilon>0$

$$
\{x \in \mathbb{C}:|x|<\epsilon, \Re x>h(\Im x)\} \subset\{x \in A:|x|<\epsilon\} .
$$

This criterion follows from Theorems 5.2 and 5.3 of Burdzy (1986b) although the Lipschitz function considered in these theorems need not be strictly positive. Observe that if a Lipschitz nonnegative function $h_{1}$ satisfies (3.1) and (3.2), and $h(r)=h_{1}(r)+\min \left(r^{2}, 1\right)$ then $h$ is Lipschitz, satisfies (3.1) and (3.2), and $h(r)>0$ for $r \neq 0$.

(2) For $a \in(-\pi / 2, \pi / 2)$, the ray $G(0, a, a, \infty)$ is not minimal thin in $D_{*}$ at 0 (see Doob (1984) 1.XII.12 Example (b)).

(3) Let $D=\{x \in \mathbb{C}:|x+1|>1\}$ and $f: D \rightarrow D_{*}$ be a conformal, oneto-one mapping onto $D_{*}$. It is easy to see that $f(D)$ is a minimal fine neighborhood of 0 in $D_{*}$, by using criterion (1) and an explicit formula for $f$. By the conformal invariance of the minimal fine topology, $D_{*}$ is a minimal fine neighborhood of 0 in $D$. Theorem 15 of Naim (1957) implies that a subset $A$ of $D_{*}$ is minimal thin in $D_{*}$ at 0 if and only if it is minimal thin in $D$ at 0 . In particular, for $a \in(-\pi / 2, \pi / 2)$, the ray $G(0, a, a, \infty)$ is not minimal thin in $D$ at 0 . Similar statements hold for the complements of other discs. 
(4) Let $D$ be the interior of $G(0,-a, a, \infty)$ for some $a \in(0, \pi)$. The function $f(x)=x^{2 a / \pi}$ defines a conformal, one-to-one mapping of $D_{*}$ onto $D$ and maps rays onto rays. It follows that for $b \in(-a, a)$, the ray $C(0, b, b, \infty)$ is not minimal thin in $D$ at 0 .

(5) Let $D=\mathbb{C} \backslash\left(\mathbb{R}_{+} \cup\{0\}\right)$ and recall the definition of $C^{+}(h, \epsilon)$ from Section 2. Assume that $h$ is Lipschitz, $h(0)=0, h \geq 0$. Let $f: D \rightarrow D_{*}, f(x)=$ $(-x)^{1 / 2}, A=f\left(C^{+}(h, \epsilon)\right)$.

Suppose first that $\limsup _{r \rightarrow 0^{+}} h(r) / r=c>0$. Then it is easy to see that $\int_{0}^{1} h(r) r^{-2} d r=\infty$. For each $\epsilon_{1}>0$, there exists $x \in A,|x|<\epsilon_{1}$, such that $\arg x=c_{1}$ for some fixed $c_{1} \in(-\pi / 2, \pi / 2)$. This implies that condition (3.1) cannot be satisfied for $A$ and, therefore, $A$ is not minimal thin in $D_{*}$ at 0 . Consequently, $C^{+}(h, \epsilon)$ is not minimal thin in $D$ at 0 .

Now consider the case when $\lim _{r \rightarrow 0^{+}} h(r) / r=0$. If $\arg x=a$ then $\arg f^{\prime}(x)=-\pi / 2+a / 2$. The graph of $h$ is a curve in $D$ approaching the real axis tangentially. Thus arg $f^{\prime}(x)$ converges to $-\pi / 2$ when $x$ converges to 0 along the graph of $h$. It follows that asymptotically, near 0 , the image of the graph of $h$ by $f$ is rotated by $-\pi / 2$ and, therefore it is a graph of a Lipschitz function $h_{1}$ i.e., for some $\epsilon_{2}>0$,

$$
\left\{x \in A:|x|<\epsilon_{2}\right\}=\left\{x \in \mathbb{C}:|x|<\epsilon_{2}, \Im x<0,0<\Re x<h_{1}(\Im x)\right\} .
$$

The "area element" $h(r) r^{-2} d r$ is mapped by $f$ approximately onto $h_{1}(\sqrt{r}) r^{-1}(d r$. $\left.r^{-1 / 2} / 2\right)$. Thus $\int_{0}^{1} h(r) r^{-2} d r<\infty$ if and only if $\int_{0}^{1} h_{1}(\sqrt{r}) r^{-3 / 2} d r<\infty$. The last integral is finite if and only if $\int_{0}^{1} h_{1}(u) u^{-2} d u<\infty$ (change the variable: $\sqrt{r}=u$ ). By criterion (1), $A$ is minimal thin in $D_{*}$ at 0 if and only if $\int_{0}^{1} h_{1}(u) u^{-2} d u<\infty$, which is equivalent to $\int_{0}^{1} h(r) r^{-2} d r<\infty$. This is also equivalent to minimal thinness of $C^{+}(h, \epsilon)$ in $D$ at 0 . Similar results hold for $C^{-}(h, \epsilon)$ and $C(h, \epsilon)$. The function $h$ in $C^{+}(h, \epsilon)$ may be assumed monotone, instead of being Lipschitz (see Burdzy (1987a)).

Geometric definitions of prime ends may be found in Pommerenke (1975) or Ohtsuka (1970). A point $x \in \mathbb{C}$ will be called a principal point of a prime end $y \in \partial_{1}^{M} D$ if $x$ is a cluster point of every continuous line in $D$ converging to $y$ in the Martin topology. A prime end $y \in \partial_{1}^{M} D$ will be called accessible if there exists a continuous line in $D$ which converges to $y$ in the Martin topology and to some point $x \in \mathbb{C}$ in the Euclidean topology. Every accessible prime end has one and only one principal point.

Suppose that $D \subset \mathbb{C}$ is simply connected and an accessible prime end $y \in \partial_{1}^{M} D$ has a principal point $x \in \mathbb{C}$. It follows from a theorem of Jackson (1980) that if $z \rightarrow y$ in the minimal fine topology then $z \rightarrow x$ in the Euclidean topology, $z \in D$. Suppose that $A, B \subset D, A$ is a minimal fine neighborhood of $y$ in $D, U \subset \mathbb{C}$ is a Euclidean neighborhood of $x$, and $A \cap U \subset B \cap U$. Then $B$ is a minimal fine neighborhood of $y$ in $D$.

Suppose that $f: \mathbb{R}_{+} \cup\{0\} \rightarrow \mathbb{C}$ is continuous and let $D$ be the unbounded connected component of $\mathbb{C} \backslash f([0, t])$, for some $t>0$ such that $f(t) \neq f(0)$. Then $D$ is open and simply connected. It is easy to prove that every analytic, oneto-one mapping of $\{x \in \mathbb{C}:|x|<1\}$ onto $D$ may be extended continuously to $\{x \in \mathbb{C}:|x| \leq 1\}$. This implies that every sequence in $D$, converging in the Martin topology converges also in the Euclidean topology. Thus every point in the minimal 
Martin boundary of $D$ corresponds to a single Euclidean boundary point (but not vice versa: one point of $\partial D$ may correspond to many different Martin boundary points). It is now obvious that all points of $\partial_{1}^{M} D$ are accessible. W.p.1, for all $t>0, F_{t}$ has similar properties.

The interior $S(a)$ of $G(0,-a, a, \infty), a \in(0, \pi / 2)$ is usually called a Stolz angle. A point $x$ will be called the angular limit of a function $f: D_{*} \rightarrow \mathbb{C}$ at $y$ and denoted $f(y)$ if $\lim _{\substack{z \rightarrow y \in S(a) \\ z-y \in y}} f(z)=x$ for every $a<\pi / 2$. The limit is taken in the Euclidean or Martin topology depending on whether $x \in \mathbb{C}$ or $x \in \partial^{M} f\left(D_{*}\right)$. For a set $A \subset \partial D_{*}, f(A)$ may have two different meanings; it may be a subset of $\mathbb{C}$ or $\partial^{M} f\left(D_{*}\right)$. For an analytic function $f: D \rightarrow \mathbb{C}$, the symbol $\mathrm{mf}-f^{\prime}(y)$ will stand for the minimal fine limit of the function $f^{\prime}$ at $y \in \partial_{1}^{M} D$ (if it exists).

Suppose that $f: D_{*} \rightarrow D, f$ is analytic, one-to-one and onto. Call a point $x \in \partial D_{*}$ an $f$-twist point if $f(x) \in \mathbb{C}$ exists and for every continuous path $\Gamma=$ $\{\Gamma(s), s \in(0,1)\} \subset D_{*}$ with $\lim _{s \rightarrow 0} \Gamma(s)=x$ one has

$$
-\liminf _{s \rightarrow 0^{+}} \arg (f(\Gamma(s))-f(x))=\limsup _{s \rightarrow 0^{+}} \arg (f(\Gamma(s))-f(x))=\infty .
$$

It is easy to see that if $x$ is an $f$-twist point then $f(x)$ is a twist point of $\partial D$ (see Section 2 for the definition).

The following version of a theorem of McMillan (1969) is proved in Burdzy $(1987 \mathrm{e})$ as Theorem 3.4. Let $f: D_{*} \rightarrow D$ be one-to-one, onto and analytic. Then for almost every $x \in \partial D_{*}$, either mf- $f^{\prime}(x) \in \mathbb{C} \backslash\{0\}$ or $x$ is an $f$-twist point.

iii) Probability. A) Various probability structures will be used in the paper. There is no need to specify them here. Unless stated otherwise, $X$ will be the canonical process on the probability space $\left\{\Omega, \mathcal{F}, P^{0}\right\}$ introduced in this subsection. In particular, the phrase "w.p.1" will usually refer to $P^{0}$.

Let $\Omega$ be the family of all functions $\omega:[0, \infty) \rightarrow \mathbb{C} \cup\{\delta\}$, continuous up to their lifetime $R=\inf \{t \geq 0: \omega(t)=\delta\}$ and constantly equal $\delta$ at $R$ and afterwards. The case $R=\infty$ is not excluded. The point $\delta$ is a "coffin" state outside $\mathbb{C}$, not identified with the usual compactification of $\mathbb{C}$. The canonical process will be denoted $X$ i.e., $X_{t}(\omega)=\omega(t)$ for all $t$ and $\omega$.

$$
\begin{gathered}
\mathcal{F}=\sigma\left\{X_{t}, t \geq 0\right\}, \\
\mathcal{F}_{t}=\sigma\left\{X_{s}, 0 \leq s \leq t\right\} .
\end{gathered}
$$

$P^{x}$ and $E^{x}$ will denote the distribution (i.e., a measure on $(\Omega, \mathcal{F})$ ) of the standard 2-dimensional Brownian motion and the corresponding expectation. The hitting time of a set $A, \inf \left\{t>0: X_{t} \in A\right\}$, will be denoted $T_{A}$.

The process

$$
Y_{t} \stackrel{\text { df }}{=} \begin{cases}X_{t} & \text { for } t<T_{D^{c}} \\ \delta & \text { otherwise }\end{cases}
$$

will be called a Brownian motion in $D$, provided $X$ has a distribution $P^{x}$ for some $x \in D$. The distribution of this process will be denoted $P_{D}^{x}$. If $P_{t}(x, d y)$ are the transition probabilities of Brownian motion in $D$ and $h$ is a positive harmonic function in $D$ then $P_{t}(x, d y) h(y) / h(x)$ are transition probabilities of a Markov process, called an $h$-process. If $h$ is a minimal harmonic function represented by $y \in \partial_{1}^{M} D$ and $X$ 
is an $h$-process starting w.p. 1 from $x \in D^{M}$ then its distribution will be denoted $P_{y}^{x}$. If $X$ has a distribution $P^{x}$ for some $x \in D$ and $P^{x}\left(X\left(T_{\partial D}\right) \in A\right)>0$ then, conditional on $\left\{X\left(T_{\partial D}\right) \in A\right\}$, the distribution of $\left\{X_{t} \mathbf{1}_{\left\{t<T_{\partial D}\right\}}+\delta \mathbf{1}_{\left\{t \geq T_{\partial D}\right\}}, t \geq 0\right\}$ is a mixture of $P_{y}^{x}$, for $y$ in a subset of $\partial_{1}^{M} D$.

B) The following version of the exit system theorem of Maisonneuve (1975) will be used in the paper. A more general probability structure than that introduced in the previous subsection will be needed.

Let $\left(\Omega, \mathcal{F}, \mathcal{F}_{t}, Y, \theta, Q\right)$ be the canonical realization of a family of Markov transition probabilities. The set $\Omega$ is the family of all right continuous functions from $[0, \infty)$ to a state space $\Gamma \cup\{\delta\}$, where $\delta$ is an isolated trap state in $\Gamma \cup\{\delta\}$. Y is the canonical process i.e., $Y_{t}(\omega)=\omega(t)$ for all $\omega$ and $t$.

$$
\begin{gathered}
\mathcal{F}^{0}=\sigma\left\{Y_{s}, s \geq 0\right\}, \\
\mathcal{F}_{t}^{0}=\sigma\left\{Y_{s}, 0 \leq s \leq t\right\} .
\end{gathered}
$$

$\mathcal{F}$ and $\mathcal{F}_{t}$ will denote the completions of $\mathcal{F}^{0}$ and $\mathcal{F}_{t}^{0}$ with respect to the family $\left\{Q^{\mu}\right\}$. Here, $Q^{\mu}$ denotes the distribution of the process with the initial distribution $\mu$. The symbol $\theta$ will stand for the usual shift operator.

Suppose that $N$ is a closed random set, homogeneous in $(0, \infty)$ i.e., for each $\omega \in \Omega, N(\omega)$ is a closed subset of $[0, \infty)$ and

$$
(N-t) \cap(0, \infty)=\left(N \circ \theta_{t}\right) \cap(0, \infty)
$$

for all $\omega$ and $t$. For each $t \geq 0$ such that $t \in N$ define an excursion $e_{t}$ of $Y$ from $N$ by

$$
e_{t}(s)= \begin{cases}Y(t+s) & \text { for } t+s<\inf \left\{u>t: Y_{u} \in N\right\} \\ \delta & \text { otherwise. }\end{cases}
$$

It is possible that $e_{t} \equiv \delta$.

Then, there exists a universally measurable family $\left\{H^{x}, x \in \Gamma\right\}$ of $\sigma$-finite measures on $\Omega$ and an additive functional $L$ such that

$$
E \cdot\left(\sum_{t \in N} Z_{t} f\left(e_{t}\right)\right)=E \cdot\left(\int_{0}^{\infty} Z_{t} H^{Y_{t}}(f) d L_{t}\right)
$$

for all positive well measurable $Z$ and all positive $\mathcal{F}$-measurable $f$, vanishing on excursions constantly equal to $\delta$. For each $x \in \Gamma, H^{x}\left(Y_{0} \neq x\right)=0$ and $\left\{Y_{t}, t>0\right\}$ is strong Markov under $H^{x}$ with the $Q$-transition probabilities, killed at the "hitting time" of $N, \inf \{t>0: t \in N\}$. The measures $H^{x}$ will be called excursion laws.

Every pair $(d L, H)$ satisfying the above properties will be called an exit system of $Y$ from $N$.

C) A special kind of excursion laws will play the central role in the paper. Return to the space $(\Omega, \mathcal{F})$ defined in subsection $(\mathrm{A})$.

An excursion law $H^{x}$ of Brownian motion in an open set $D \subset \mathbb{C}$ is a $\sigma$-finite measure on $\Omega$ such that $\left\{X_{t}, t>0\right\}$ is strong Markov under $H^{x}$ with the $P_{D}$ transition probabilities and $H^{x}\left(\lim _{t \rightarrow 0} X_{t} \neq x\right)=0$. An excursion law $H^{x}$ will be called standard if $0<H^{x}\left(T_{B}<\infty\right)<\infty$ for every nonpolar compact set $B \subset D$. The point $x$ may be an element of the Euclidean or the minimal Martin boundary. 
A point $x \in \partial_{1}^{M} D$ will be called attainable if $P_{x}^{y}(R<\infty)=1$ for some (and therefore all) $y \in D$. If $D$ is simply connected and $x \in \partial_{1}^{M} D$ is accessible then it is attainable (Burdzy $(1987 \mathrm{c}, \mathrm{d})$ ). For each attainable $x \in \partial_{1}^{M} D$ there exists a unique, up to a multiplicative constant, standard excursion law $H^{x}$ in $D$.

A set $A$ will be called $H^{x}$-regular if $H^{x}\left(T_{A} \neq 0\right)=0$. If $x \in \partial_{1}^{M} D$ and $H^{x}$ is the standard excursion law in $D$ then $A \subset D$ is $H^{x}$-regular if and only if it is not minimal thin in $D$ at $x$ (Burdzy (1987a)). This property may be derived from another probabilistic interpretation of the minimal fine topology: a set $A \subset D$ is minimal thin in $D$ at $x \in \partial_{1}^{M} D$ if and only if the last exit time of $X$ from $A$ is strictly less than the lifetime $R, P_{x}^{y}$-a.s. for every $y \in D$ (Doob (1984) 3.III.3).

Suppose that $H_{1}^{x}$ is a standard excursion law in $D_{1}, f: D_{1} \rightarrow D_{2}$ is one-to-one, analytic and onto and $f(x)$ is an accessible prime end. Denote

$$
c(t)=\int_{0}^{t}\left|f^{\prime}(X(s))\right|^{2} d s \text { for } t \in(0, R)
$$

and

$$
Y(c(t))= \begin{cases}f(X(t)) & \text { if } t \in(0, R) \text { and } c(t)<\infty \\ \delta & \text { otherwise. }\end{cases}
$$

If the distribution of $X$ is $H_{1}^{x}$ then the distribution of $Y$ is a standard excursion law in $D_{2}$, denoted $f\left(H_{1}^{x}\right)$.

Now, consider a special case. Let $H_{*}^{0}$ be the standard excursion law in $D_{*}, f(x)=$ $x^{a}$ for some $a \in(1,2)$, and $D=f\left(D_{*}\right)$. Then $f\left(H_{*}^{0}\right) \stackrel{\text { df }}{=} H^{0}$ is a standard excursion law in D. By Theorem 3.3 (iii) of Burdzy (1986a),

$$
H_{*}^{0}\left(\max _{t \in(0, R)}\left|X_{t}\right| \in d x\right)=c x^{-2} d x
$$

so

$$
H^{0}\left(\max _{t \in(0, R)}\left|X_{t}\right| \in d x\right)=c_{1} x^{-1-1 / a} d x
$$

and

$$
H^{0}\left(\max _{t \in(0, R)}\left|X_{t}\right|>\epsilon\right)<\infty \text { for } \epsilon>0 .
$$

If $X$ has the distribution $H^{0}$ then $\left\{c_{2} X\left(t / c_{2}^{2}\right), t \geq 0\right\}$ is also a standard excursion law in $D$, since a similar scaling property characterizes Brownian motion. By the uniqueness of the standard excursion law, $H_{1}^{0}=c_{3} H^{0}$. This and (3.4) imply that $c_{3}=c_{2}^{1 / a}$. This, in turn, may be used to derive the following formula: $H^{0}(R \in$ $d t)=c_{4} t^{-1-1 /(2 a)} d t$. In particular, the expectations of $\max _{s \in(0, R)}|X(s)|$ and $R$ are finite under $H^{0}$.

D) Let $\lambda$ be the Lebesgue measure on $\mathbb{R}_{+}$and $\mu$ be a $\sigma$-finite measure on a set $U$. Then there exists a Poisson point process $Z$ on $\mathbb{R}_{+} \times U$ with the characteristic measure $\lambda \times \mu$ i.e., $Z$ is a random subset of $\mathbb{R}_{+} \times U$ defined on some probability space, such that for every pair $A, B$ of disjoint, nonrandom subsets of $\mathbb{R}_{+} \times U$, $\operatorname{card}(Z \cap A)$ and $\operatorname{card}(Z \cap B)$ are independent and have Poisson distributions with mean values $(\lambda \times \mu)(A)$ and $(\lambda \times \mu)(B)$. W.p.1 there are no two points in $Z$ with the same first coordinate (Itô (1972)). 


\section{Path behavior near $V_{t}$.}

Proof of Theorem 2.2. For a random variable $Y_{0}$ define $Y_{t}=\sup \left(Y_{0}, V_{t}\right)$. Let $Q^{x, y}$ be the distribution of $\left\{\left(X_{t}, Y_{t}\right), t \geq 0\right\}$ when $Y_{0} \equiv y$ and $X$ has the distribution $P^{x}$. It is easy to see that the process $(X, Y)$ under $Q^{x, y}$ is strong Markov with the transition probabilities $Q$. From now on, it will be assumed that $(X, Y)$ is the canonical realization of the family of transition probabilities $Q$, in the sense of Section 3(iii)(B).

Let $N=\left\{t \geq 0: X_{t}=Y_{t}\right\}$. Then $N$ is a homogeneous closed random set and there exists an exit system $(d L, H)$ of $(X, Y)$ from $N$. Choose a compact nonpolar set $B, B \subset D \stackrel{\text { df }}{=} \mathbb{C} \backslash\left(\mathbb{R}_{+} \cup\{0\}\right)$ and apply the exit system formula (3.3) with $T=\inf \left\{t \geq 0: X_{t} \in B+Y_{t}\right\}, f\left(e_{t}\right)=\mathbf{1}_{\{T<\infty\}}\left(e_{t}\right), Z_{t}=\mathbf{1}_{[0, T]}(t)$ to obtain

$$
E^{\cdot}(1)=E^{\cdot}\left(\int_{0}^{T} H^{X_{t}, Y_{t}}(T<\infty) d L_{t}\right) .
$$

Thus the right hand side is finite and, therefore, $d L$ does not charge w.p.1 the set $\left\{t \leq T:\left(X_{t}, Y_{t}\right) \in\left\{(x, y): H^{x, y}(T<\infty)=\infty\right\}\right\}$. By a similar argument applied with $T$ replaced by consecutive hitting times of $B+Y_{t}$ by $X_{t}$, one can show that the measure $d L$ does not charge $\left\{t<\infty:\left(X_{t}, Y_{t}\right) \in\left\{(x, y): H^{x, y}(T<\infty)=\infty\right\}\right.$. This shows that the measures $H^{x, y}$ with $H^{x, y}(T<\infty)=\infty$ play no role in the exit system (3.3), i.e. the value of the right hand side of (3.3) would not change if all such measures were replaced by null measures. Thus, it may be assumed that $H^{x, y}(T<\infty)<\infty$ for all $x$ and $y$. The only excursion laws $H^{x, y}$ that count in (3.3) are such that $x=y \in \mathbb{R}_{+}$since w.p.1 there are no excursions of $(X, Y)$ from $N$ starting from points $(x, y)$ with $x \neq y$. For $x \in \mathbb{R}_{+}$, the process $\left\{\left(X_{t}, Y_{t}\right), t>0\right\}$ under $H^{x, x}$ is strong Markov with the transition probabilities $Q$, killed at

$$
T_{x} \stackrel{\text { df }}{=} \inf \left\{t>0: \Im X_{t}=0, \Re X_{t} \geq x\right\}
$$

and $H^{x, x}\left(\left(X_{0}, Y_{0}\right) \neq(x, x)\right)=0$. It follows that under $H^{x, x}, Y$ is equal to $x$ up to its lifetime and $X$ is strong Markov with the transition probabilities $P_{D+x}$. Let $H_{1}^{x}$ denote the distribution of $X$ under $H^{x, x}, x \in \mathbb{R}_{+}$. This measure is an excursion law of Brownian motion in $D+x$. By the argument given above, $H_{1}^{x}\left(T_{B+x}<\infty\right)=$ $H^{x, x}(T<\infty)<\infty$ for every compact set $B \subset D$. One has $H_{1}^{x}\left(T_{B+x}<\infty\right)>0$ because otherwise the measure would have to be null (which is possible but such measures play no role in the proof). It follows that it may be assumed that all measures $H_{1}^{x}$ are standard. Thus, a subset of $D+x$ is $H_{1}^{x}$-regular if and only if it is not minimal thin in $D+x$ at $x$.

Suppose that $\int_{0}^{1} h(r) r^{-2} d r=\infty$. Then $x+C^{+}(h, \epsilon), \epsilon>0$, is not minimal thin in $D+x$ at $x$ (see Section 3 (ii)), and it is $H_{1}^{x}$-regular. Therefore, $\left(x+C^{+}(h, \epsilon)\right) \times \mathbb{R}$ is $H^{x, x}$-regular. The exit system formula (3.3) implies that $Q^{0,0}$-a.s., every excursion $e_{t}=\left(e_{t}^{1}, e_{t}^{2}\right)$ of $(X, Y)$ from $N$ hits immediately every set $\left(e_{t}^{1}(0)+C^{+}(h, \epsilon)\right) \times \mathbb{R}$, for all rational $\epsilon>0$. Observe that for a fixed $t>0, \Im X_{t} \neq 0$ w.p.1, so $V_{t}$ corresponds to the starting point of the last excursion of $(X, Y)$ from $N$ before $t$. Thus, for a fixed $t>0$, w.p.1 $\left(V_{t}+C^{+}(h, \epsilon)\right) \cap A_{t} \neq \emptyset$ for every rational $\epsilon>0$. This completes the proof of Theorem 2.2 (ii).

Now assume that $\int_{0}^{1} h(r) r^{-2} d r<\infty$. In this case, $(x+C(h, 1)) \times \mathbb{R}$ is not $H^{x, x}$-regular. The exit system formula implies that $Q^{0,0}$-a.s., for each excursion 
$e_{t}=\left(e_{t}^{1}, e_{t}^{2}\right)$ of $(X, Y)$ from $N$ there exists some $\epsilon>0$ such that $\left(e_{t}^{1}(0)+C(h, \epsilon)\right) \times \mathbb{R}$ is not immediately hit by $\left\{e_{t}(s), s>0\right\}$. It follows that for a fixed $t>0$, w.p.1 there exists $\epsilon>0$ such that

$$
X_{u} \notin V_{t}+C(h, \epsilon) \text { for } u \in(S, t),
$$

where $S=\sup \left\{s<t: X_{s}=V_{t}\right\}$.

It is easy to see that $H_{1}^{x}(X(R-)=x)=0$, so the exit system formula implies that $Q^{0,0}$-a.s. all excursions $e_{t}=\left(e_{t}^{1}, e_{t}^{2}\right)$ of $(X, Y)$ from $N$ have the property $e_{t}^{1}(0) \neq e_{t}^{1}(R-)$, where $R$ is the lifetime of $e_{t}$. Thus, w.p. 1 there are no $s, t, u, 0<$ $s<t$, such that $X_{s}=X_{t}=V_{u}$ and, therefore, $S=\inf \left\{s>0: X_{s}=V_{t}\right\}$ w.p.1.

Let $U(s)=T_{\mathbb{R}_{+}}+S$. The strong Markov property applied at $T_{\mathbb{R}_{+}}$shows that for every $\mu, P^{\mu}$-a.s., for every rational $s>0$ there exists $\epsilon>0$ such that $X_{u} \notin$ $V_{U(s)}+C(h, \epsilon)$ for $u<U(s), u>\inf \left\{v>0: X_{v}=V_{U(s)}\right\}$. Observe that for a fixed $t>0, P^{\mu}$-a.s., either $T_{\mathbb{R}_{+}}>t$ or $V_{t}=V_{U(s)}$ for some rational $s>0$ such that $U(s)>t$. Thus for a fixed $t>0, P^{\mu}$-a.s. either $T_{\mathbb{R}_{+}}>t$ or there exists $\epsilon>0$ such that

$$
X_{u} \notin V_{t}+C(h, \epsilon) \text { for } u \in(S, t] \text {. }
$$

Now, let $\mu$ be a normal distribution, say $\mu(d x)=P^{0}\left(X_{u} \in d x\right)$ for some $u>0$. Then the distributions of $\left\{X_{s}, s \in(0, t)\right\}$ and $\left\{W_{s} \stackrel{\text { df }}{=} X_{t-s}, s \in(0, t)\right\}$ are mutually absolutely continuous, provided $X$ has the distribution $P^{\mu}$. It follows that for a fixed $t>0, P^{\mu}$-a.s., either $T_{\mathbb{R}_{+}}>t$ or there exists $\epsilon>0$ such that

$$
X_{u} \notin V_{t}+C(h, \epsilon) \text { for } u \in[0, S)
$$

since $V_{t}={ }^{W} V_{t}$ and, $P^{\mu}$-a.s.,

$$
S=\inf \left\{s>0: X_{s}=V_{t}\right\}=\sup \left\{s<t: X_{s}=V_{t}\right\}
$$

This, combined with (4.1), proves that for a fixed $t>0, P^{\mu}$-a.s., either $T_{\mathbb{R}_{+}}>t$ or there exists $\epsilon>0$ such that $V_{t}+C(h, \epsilon) \subset F_{t}$. It follows that for a fixed $t>0$, $P^{0}$-a.s., for all rational $s \in(0, t)$, either $\inf \left\{u>0: W_{u}^{s} \in \mathbb{R}_{+}\right\}>t$ or there exists $\epsilon>0$ such that

$$
{ }^{W^{s}} V_{t}+C(h, \epsilon) \subset{ }^{W^{s}} F_{t},
$$

where $W_{u}^{s}=X_{s+u}$. The continuity of paths implies now easily that for a fixed $t>0, P^{0}$-a.s. there exists $\epsilon>0$ such that $V_{t}+C(h, \epsilon) \subset F_{t}$. This completes the proof of Theorem 2.2 (i).

Proof of theorem 2.1. i) Fix $t>0$. Obviously, $G\left(V_{t}, 0,0,1\right) \subset F_{t}$. Observe that $C^{+}(h, \epsilon)+V_{t}=G\left(V_{t}, 0, a, \epsilon\right)$ for $h(r)=r \tan a, a \in(0, \pi / 2)$. Since $\int_{0}^{1}(r \tan a) r^{-2} d r=$ $\infty$, Theorem 2.2 (ii) implies that w.p.1 $V_{t}+C^{+}(h, \epsilon)=G\left(V_{t}, 0, a, \epsilon\right)$ intersects $A_{t}$ for every $\epsilon>0$. The same holds for $G\left(V_{t}, a, 0, \epsilon\right), a \in(-\pi / 2,0)$, by symmetry. It follows that $V_{t} \in B_{t}^{F}(0,0)$.

ii) The main part of the proof of Theorem 2.1 (ii)-(iv) will be based on an idea dicovered independently by El Bachir (1983) and Le Gall (1986). Readers are referred to Le Gall (1987) for the details (El Bachir's thesis contains an error). 
Fix $a, b, a \in(-\pi, 0), b \in(0, \pi)$. Let $W_{t}=\inf \left\{x \in \mathbb{R}_{+}: G(x, a, b, \infty) \cap A_{t}=\emptyset\right\}$. Consider the process $\left\{Y_{t}, t \geq 0\right\}, Y_{t} \stackrel{\mathrm{df}}{=} X_{t}-W_{t}$. If $X$ has a distribution $P^{x}$ for some $x \in \mathbb{C}, \Im x>0$, then, up to the stopping time $T_{\mathbb{R}}, Y$ is a reflected Brownian motion in $\mathbb{C} \backslash(G(0,-\pi, b, \infty) \cup\{0\})$ with the oblique reflection on $\partial G(0,-\pi, b, \infty)$. This follows from Theorem 6.1 of Le Gall (1987). Moreover, it has been shown that the vector of reflection is parallel to the real axis. It follows that $Y$ is a reflected Brownian motion in $D \stackrel{\text { df }}{=} \mathbb{C} \backslash(G(0, a, b, \infty) \cup\{0\})$, with the vector of reflection parallel to $\mathbb{R}$, on both sides of the wedge $D$. In the notation and conventions of Varadhan and Williams (1985), the process $Y$ is a reflected Brownian motion in a wedge with angle $2 \pi-(b-a)$ and angles of reflection $\theta_{1}=\pi / 2-b$ and $\theta_{2}=\pi / 2+a$. Varadhan and Williams (1985) have shown that the process $Y$ reaches 0 if and only if $\theta_{1}+\theta_{2}>0$, i.e., $b-a<\pi$. It follows easily that, when $X$ has the distribution $P^{0}$, then w.p.1, $Y_{t}=0$ for some $t>0$ if and only if $b-a<\pi$.

Fix $a, b, a \in(-\pi, 0), b \in(0, \pi), b-a \geq \pi$. W.p.1, there is no $t>0$ such that $G\left(V_{t}, a, b, \infty\right) \subset F_{t}$. It follows that w.p.1, there are no rational $s>0$ and arbitrary $t>0$ such that $G\left(Z^{s} V_{t}, a, b, \infty\right) \subset Z^{s} F_{t}$, where $Z_{t}^{s}=X_{t+s}$. If $V_{t} \in B_{t}^{F}(a, b)$ then $G\left(Z^{s} V_{u}, a, b, \infty\right) \subset{ }^{s} F_{u}$ for some rational $s \in(0, t)$ and $u>0$. This implies that w.p.1, there is no $t>0$ such that $V_{t} \in B_{t}^{F}(a, b)$. In particular, Theorem 2.1 (iii) holds true.

W.p.1, there are no rational $a \in(-\pi, 0), b \in(0, \pi), b-a<\pi$, rational $s>0$ and arbitrary $u>0$ such that $G\left({ }^{Z^{s}} V_{u}, a, b, \infty\right) \subset{ }^{s} F_{u}$. If $V_{t} \in B_{t}^{F}(a, b)$ for some $t>0$ and $a \in(-\pi, 0), b \in(0, \pi), b-a>\pi$, then there exist rational $a_{1} \in(-\pi, 0), b_{1} \in$ $(0, \pi), a_{1}>a, b_{1}<b, b_{1}-a_{1}>\pi, s>0$ and $u>0$ such that $G\left({ }^{s} V_{u}, a_{1}, b_{1}, \infty\right) \subset$ $Z^{s} F_{u}$. Thus, w.p. 1 there are no $a \in(-\pi, 0), b \in(0, \pi), b-a>\pi$ and $t>0$ such that $V_{t} \in B_{t}^{F}(a, b)$. In other words, Theorem 2.1 (iv) holds.

Now suppose that $a<0, b>0, b-a<\pi$. Denote $T_{x}=\inf \left\{t>0: X_{t} \in\right.$ $\partial G(x, a, b, \infty)\}$. In the present case, the point 0 is a regular point for the process $Y$ (Williams (1987)), so the set $\left\{t<T_{1}: G\left(X_{t}, a, b, \infty\right) \subset F_{t}, X_{t} \in \mathbb{R}_{+}\right\}$is nonempty w.p.1. Denote its maximum $T$. If $a$ and $b$ are fixed then each point $x \in \mathbb{C}$ lies on the boundary of exactly one set $G(y, a, b, \infty), y \in \mathbb{R}$. Let $h_{x}$ denote the minimal harmonic function in $D_{y} \stackrel{\text { df }}{=} \mathbb{C} \backslash(G(y, a, b, \infty) \cup\{0\})$, represented by $x$ (here $x \in \partial D_{y}$ ).

Fix some $y \in \mathbb{R}_{+}, y<1$. Consider an event $\left\{X_{T} \in[x, x+\Delta x]\right\}$, for some $\Delta x>0,[x, x+\Delta x] \subset[y, 1]$. By the strong Markov property, the process $\left\{X_{t}, 0<\right.$ $\left.t<T_{x}\right\}$ is independent of $T$ given $\left\{X_{T} \in[x, x+\Delta x]\right\}$ and $\left\{X\left(T_{x}\right)=z\right\}$. Thus, given $\left\{X_{T} \in[x, x+\Delta x]\right\}$ and $\left\{X\left(T_{x}\right)=z\right\}$ the process $\left\{X_{t}, 0<t<T_{x}\right\}$ is a Brownian motion conditioned to be $z$ at $T_{x}$; in other words, it is an $h_{z}$-process in $D_{x}$. It follows that given $\left\{X_{T} \in[x, x+\Delta x]\right\}$ and $\left\{X\left(T_{x}\right)=z\right\}$, the process $\left\{X_{t}, 0<t<T_{y}\right\}$ is an $h_{z}$-process in $D_{y}$ (Doob (1984) 2.X.2).

Observe that when $\Delta x \rightarrow 0$ then the conditional distribution of $X\left(T_{x}\right)-x$ given $\left\{X_{T} \in[x, x+\Delta x]\right\}$ converges to the point mass at 0 , uniformly in $x \in[y, 1]$. Easy explicit computations show that $h_{z}$ converges to $h_{x}$, uniformly on compacts in $D_{y}$, when $z \rightarrow x \notin D_{y}$. The last two observations permit to conclude that given $\left\{X_{T}=x\right\}, x \in[y, 1]$, the distribution of $\left\{X_{t}, 0<t<T_{y}\right\}$ is that of an $h_{x}$-process in $D_{y}$. Now fix $x$ and let $y \rightarrow x$. It follows that the distribution of $\left\{X_{t}, 0<t<T\right\}$ given $\left\{X_{T}=x\right\}$ is that of an $h_{x}$-process in $D_{x}$.

For $c \in(-\pi, a) \cup(b, \pi)$ the set $G(x, c, c, \infty)$ is not minimal thin in $D_{x}$ at $x$. It follows that for every such $c$, w.p. $1 \sup \left\{t<T: \arg \left(X_{t}-X_{T}\right)=c\right\}=T$ (see Section 3 (iii) (C)). Thus, w.p. 1 there are no $a_{1} \leq a, b_{1} \geq b, b_{1}-a_{1}>b-a, \epsilon_{1}>0$ such 
that $G\left(X_{T}, a_{1}, b_{1}, \epsilon_{1}\right) \subset F_{T}$. This, combined with the definition of $T$, shows that $X_{T}=V_{T} \in B_{T}^{F}(a, b)$. This completes the proof of Theorem 2.1 (ii).

\section{Path behavior near $M_{t}$.}

Proof of Theorem 2.3. i) The proof will be only sketched because it is completely analogous to that of Theorem 2.2.

By abuse of notation, $M_{t}$ will denote its element, in the case it contains only one element.

For a random variable $Y_{0}$ define

$$
Y_{t}=\min \left(Y_{0}, \inf \left\{\left|X_{s}-1\right|: s \in[0, t]\right\}\right) .
$$

Let $Q^{x, y}$ be the distribution of $\left\{\left(X_{t}, Y_{t}\right), t \geq 0\right\}$ when $Y_{0} \equiv y$ and $X$ has the distribution $P^{x}$. The process $(X, Y)$ under $Q^{x, y}$ is strong Markov with the transition probabilities $Q$. It may be assumed that $(X, Y)$ is the canonical realization of the family of transition probabilities $Q$. Let $N=\left\{t \geq 0:\left|X_{t}-1\right|=Y_{t}\right\}$ and let $(d L, H)$ be an exit system of $(X, Y)$ from $N$. The only excursion laws $H^{x, y}$ that count are such that $|x-1|=y$. Let $H_{1}^{x}$ be the distribution of $X$ under $H^{x,|x-1|}$. Then $H_{1}^{x}$ is a standard excursion law in $D_{x} \stackrel{\text { df }}{=}\{z \in \mathbb{C}:|z-1|>|x-1|\}$.

Every half-line $K_{x}$ with endpoint $x$, not intersecting $\mathbb{C} \backslash D_{x}$ is not minimal thin in $D_{x}$ at $x$ so it is $H_{1}^{x}$-regular. Thus, $Q^{0,1}$-a.s., every excursion $e_{t}=\left(e_{t}^{1}, e_{t}^{2}\right)$ of $(X, Y)$ from $N$ hits immediately every set $K_{x} \times \mathbb{R}_{+}$, where $x=e_{t}^{1}(0)$ and $K_{x} \cap\left(\mathbb{C} \backslash D_{x}\right)=\emptyset$. It follows that for a fixed $t>0$, w.p.1 for all $a, b, b-a>\pi, \epsilon>0$, one has $G\left(M_{t}, a, b, \epsilon\right) \cap A_{t} \neq \emptyset$.

Let $U_{x}$ be the half-space containing $\{z \in \mathbb{C}:|z-1| \leq|x-1|\}$, such that $\partial U_{x}$ is tangent to this disc at $x$. The set $U_{x} \cap D_{x}$ is minimal thin in $D_{x}$ at $x$, so it is not $H_{1}^{x}$-regular. As in the proof of Theorem 2.2, one can show that for a fixed $t>0$, w.p. 1 there exists $\epsilon>0$ and $a, b, b-a=\pi$, such that

$$
X_{u} \notin G\left(M_{t}, a, b, \epsilon\right) \text { for } u \in(S, t],
$$

where $S=\sup \left\{s>0: X_{s} \in M_{t}\right\}$. The time reversal trick shows that for each $t>0$, w.p. 1 there exist $\epsilon_{1}>0$ and $a, b, b-a=\pi$ such that $X_{s} \notin G\left(M_{t}, a, b, \epsilon\right)$ for $s \in[0, t]$. Thus, for $t>0$, w.p. $1 M_{t} \in B_{t}(\pi)$.

ii) Fix $a, b \in \mathbb{R}, b-a \in(\pi, 3 \pi / 2)$. The results of Le Gall (1987) show that w.p.1 there exists $t>0$ such that $X_{t} \in B_{t}^{F}(a, b)$. This implies easily that for some $p>0$,

$$
P^{x}\left(\left\{X_{t}\right\}=M_{t} \subset B_{t}(a, b) \text { for some } t<T_{1 / 2}\right) \geq p
$$

for all $|x-1|=1$, where

$$
T_{r}=\inf \left\{t>0:\left|X_{t}-1\right| \leq r\right\}
$$

By scaling,

$$
P^{x}\left(\left\{X_{t}\right\}=M_{t} \subset B_{t}(a, b) \text { for some } t<T_{2^{-k}}\right) \geq p
$$

for all $|x-1|=2^{-k+1}, k \geq 1$. The events $\left(\left\{X_{t}\right\}=M_{t} \subset B_{t}(a, b)\right.$ for some $\left.t \in\left(T_{2^{-k+1}}, T_{2^{-k}}\right)\right)$ have $P^{0}$-probabilities greater or equal to $p$, by the strong Markov property. It follows that infinitely many of these events will happen, with probability not less than $p$. Since such an event belongs to the tail $\sigma$-field and $p>0$, it is certain. Theorem 2.3 (ii) has been proved. 


\section{Existence of cone points.}

Proof of Theorem 2.4. i) The proof will consist of several steps.

(A) The case $b-a \in(0, \pi)$. Construction of a 2-dimensional Brownian motion $Y$ from excursions in a wedge.

(B) Paths of $Y$ contain cone points.

(C) The set of cone points is dense.

(D) Same as (C), for $b-a=\pi$.

(E) Same as (C), for $b-a=0$.

(F) Proof of the theorem for floating $t$ ( $t$ has been fixed in $(A)-(E)$ ).

(A) Fix $a, b, b-a \in(0, \pi)$. Since Brownian motion is rotation invariant, it is enough to consider only the case $b=-a>0$. Let $D=\mathbb{C} \backslash(G(0,-b, b, \infty) \cup\{0\})$ and $H^{0}$ be the standard excursion law in $D$. Consider a Poisson point process on $\mathbb{R}_{+} \times \Omega$ with the characteristic measure $\lambda \times H^{0}$, where $\lambda$ is Lebesgue measure. The elements of the random set $Z$ may be unambiguously denoted by $\left(t, f_{t}\right)$ (see Section 3 (iii) (D)). Denote $R_{t}=\inf \left\{s>0: f_{t}(s)=\delta\right\},\left|f_{t}\right|=\sup _{s \in\left(0, R_{t}\right)}\left|f_{t}(s)\right|, R_{t}^{\epsilon}=R_{t}-\epsilon$, $f_{t}^{\epsilon}(s)=f_{t}(s+\epsilon)-f_{t}(\epsilon)$. Under $H^{0}$, the lifetime $R$ and $\max _{s \in(0, R)}|X(s)|$ have densities $c_{1} t^{-1-1 /(2 a)}$ and $c_{2} x^{-1-1 / a}$, resp. (see Section 3 (iii) (C)). Theorem 4.6 of Itô (1972) shows that for every fixed $u>0$, w.p. $1 \sum_{t \leq u} R_{t}<\infty, \sum_{t \leq u}\left|f_{t}\right|<\infty$ and, therefore, $\sum_{t \leq u}\left|f_{t}\left(R_{t}-\right)\right|<\infty$. It follows that for each $\epsilon \geq 0$, w.p.1, the following process $Y^{\epsilon}$ is well defined for all $t>0$. The sums in the next two formalae will extend over $u$ such that $R_{u}^{\epsilon}>0$. First note that

$$
t= \begin{cases}s+\sum_{u<r} R_{u}^{\epsilon} & \text { if there exists }\left(r, f_{r}\right) \in Z \text { such that } R_{r}^{\epsilon}>0, \\ \sum_{u<r} R_{u}^{\epsilon} & \text { otherwise, }\end{cases}
$$

defines unique $r>0$ and $s \in\left[0, R_{r}^{\epsilon}\right)$. Then let

$$
Y_{t}^{\epsilon}= \begin{cases}f_{r}^{\epsilon}(s)+\sum_{u<r} f_{u}^{\epsilon}\left(R_{u}^{\epsilon}-\right) & \text { if there exists }\left(r, f_{r}\right) \in Z \text { such that } R_{r}^{\epsilon}>0, \\ \sum_{u<r} f_{u}^{\epsilon}\left(R_{u}^{\epsilon}-\right) & \text { otherwise, }\end{cases}
$$

and let $Y \stackrel{\text { df }}{=} Y^{0}$.

Since $H^{0}\left(\max _{t \in(0, R)}\left|X_{t}\right| \geq \epsilon_{1}\right)<\infty$ for $\epsilon_{1}>0$, it follows that for $r>0, \epsilon_{1}>0$, w.p.1 there are only a finite number of excursions $f_{u}$ with $u \leq r$ and $\left|f_{u}\right| \geq \epsilon_{1}$. This and the continuity of each function $s \rightarrow f_{t}(s)$ imply the continuity of $Y$. The continuity of the process $Y^{\epsilon}$ is even more evident, since the sums in the definition of $Y^{\epsilon}$ are finite w.p.1, for $\epsilon>0$.

For a fixed $\epsilon>0$, the processes $\left\{f_{t}^{\epsilon}(s), s \geq 0\right\}$ are independent, which follows from the strong Markov property of $Z$ (Theorem 5.1 of Itô (1972)). Consider a process $\left\{f_{t}^{\epsilon}(s), s \geq 0\right\}$ together with its natural filtration, enlarged by the sets generated by $f_{t}(\epsilon)$. By the strong Markov property of $H^{0}$, such a process is a Brownian motion killed at a stopping time. It follows that $Y^{\epsilon}$ is pieced together from such independent Brownian segments, a finite number of them on each finite time interval. It is now evident that $Y^{\epsilon}$ is a Brownian motion.

For $u>0$, w.p. $1 \sum_{t \leq u} R_{t}<\infty$ and $\sum_{t \leq u}\left|f_{t}\right|<\infty$ so it is easy to see that for a fixed $t>0$, w.p.1. $Y^{\epsilon} \rightarrow Y$ in the supremum norm on $[0, t]$. It follows that $Y$ is a Brownian motion. 
(B) If $x_{1}, x_{2}, \ldots$ belong to $D^{c}$ then $\sum_{k=1}^{\infty} x_{k} \in D^{c}$, provided the sum is well defined. This implies that

$$
D^{c}+\sum_{t \leq v<u} f_{v}\left(R_{v}-\right) \subset D^{c}
$$

and

$$
f_{t}(s) \notin D^{c}+\sum_{t \leq v<u} f_{v}\left(R_{v}-\right), \text { for } t<u, s \in\left(0, R_{t}\right) .
$$

Since $Y$ is continuous, a similar property holds for points outside excursions $f$ and it follows that for $t>0$, w.p. $1 Y_{s} \notin Y_{T}+G(0,-b, b, \infty)$ for all $s \in[0, t]$, where $T=T(t)=\sum_{s<u_{0}} R_{s}, u_{0}=u_{0}(t)=\inf \left\{u \geq 0: \sum_{s \leq u} R_{s} \geq t\right\}$. Hence, for $t>0$,w.p.1, $G\left(Y_{T},-b, b, \infty\right) \subset{ }^{Y} F_{t}$.

For each rational $c \in(-\pi,-b) \cup(b, \pi)$, the ray $G(0, c, c, \infty)$ is $H^{0}$-regular so, w.p.1, for all such $c$ and all excursions $f_{s}$ one has $\inf \left\{u>0: f_{s}(u) \in G(0, c, c, \infty)\right\}=$ 0 . The stable process $\left\{\sum_{u \leq s} R_{u}, s \geq 0\right\}$ does not hit a point fixed in advance, so for a fixed $t>0$, w.p.1, $T(t)<t$ and $Y(T)$ is the starting point of the (translated) excursion $f_{u_{0}}$. This implies that for all $a_{1}, b_{1}, a_{1} \leq-b, b_{1} \geq b, b_{1}-a_{1}>2 b, \epsilon_{1}>0$, the set $G\left(Y_{T}, a_{1}, b_{1}, \epsilon_{1}\right)$ intersects ${ }^{Y} A_{t}$. Thus, for $t>0$, w.p.1 $Y_{T} \in{ }^{Y} B_{t}^{F}(-b, b)$ and, moreover $\Re Y_{T} \geq 0$.

Let $t>0, d>0, T_{d}=\inf \left\{s>0: \Re Y_{s}=d\right\}, W^{d}(s)=Y\left(T_{d}+s\right)$. Condition on $T_{d}$ so that $t-T_{d}$ is "fixed." Then $\left\{W^{d}(s), s \in\left[0, t-T_{d}\right]\right\}$ is a Brownian motion, unless $T_{d} \geq t$. Apply to $W^{d}$ the result already proved for $Y$, to see that there exists w.p.1 $U=U(d) \in\left(0, t-T_{d}\right)$ such that $W^{d}(U) \in W^{d} B_{t}^{F}(-b, b)$ and $\Re W^{d}(U) \geq d$ (unless $T_{d} \geq t$ ). Of course, $Y\left(\left[0, T_{d}\right]\right) \subset\{x \in \mathbb{C}: \Re x \leq d\}$, so $W^{d}(U) \in{ }^{Y} B_{t}^{F}(-b, b)$. This holds w.p.1 for all rational $d$ simultaneously. It may be concluded that for a fixed $t>0$, w.p.1, for every $\epsilon>0$ there exists $x \in{ }^{Y} B_{t}^{F}(-b, b)$ such that $\Re x \geq \max _{s \in[0, t]} \Re Y_{s}-\epsilon$.

(C) Now switch to the canonical notation, i.e., let $X$ be the standard Brownian motion. Fix real numbers $p_{1}, p_{2}, p_{3}, p_{2}<p_{3}$, and denote

$$
N=\left\{x \in \mathbb{C}: \Re x \leq p_{1}, \Im x \in\left[p_{2}, p_{3}\right]\right\}
$$

It can be easily proved that w.p.1 either

$$
p_{1}=\max \left\{\Re X_{u}: u \in[0, t], X_{u} \in N\right\}
$$

or this maximum is attained at a single point of $(0, t)$, say $s$. Consider this last case.

Let $y_{k} \in \partial N$ be such that $\Im y_{k}=p_{k}, \Re y_{k}=\max \left\{\Re X_{u}: \Im X_{u}=p_{k}, u \in\right.$ $[0, t]\}, k=2,3$. The points $y_{2}$ and $y_{3}$ belong to $B_{t}(0,0)$, which may be shown as in the part of the present proof devoted to the case $b-a=0$ (see below). It follows that $X_{s} \notin \partial N$.

Choose $q_{1}$ and $q_{2}, q_{1}<q_{2}$, such that $s \in\left(q_{1}, q_{2}\right)$ and $X\left(\left[q_{1}, q_{2}\right]\right) \subset N$. Denote $W=\left\{X(u), u \in\left[q_{1}, q_{2}\right]\right\}$ and observe that w.p.1 there exists a sequence $\left\{x_{k}\right\}$ such $x_{k} \in{ }^{W} B_{t}^{F}(-b, b)$ and $\Re x_{k} \geq \max _{u \in\left[q_{1}, q_{2}\right]} \Re X_{u}-1 / k=\Re X_{s}-1 / k, k \geq 1$. The continuity of $X$ implies that $\lim _{k \rightarrow \infty} x_{k}=X_{s}$ and there exists $\epsilon>0$ such that $\left\{x \in A_{t}:\left|X_{s}-x\right|<\right.$ 
$\epsilon\}=\left\{x \in X\left(\left[q_{1}, q_{2}\right]\right):\left|X_{s}-x\right|<\epsilon\right\}$. The last two facts (see also Section 3(ii)) show that $x_{k} \in B_{t}(-b, b)$ for large $k$. Moreover, if $\left\{x \in \partial N: \Re x=p_{1}\right) \subset F_{t}$ then $x_{k} \in B_{t}^{F}(-b, b)$ for large $k$.

Consider the family of all sets $N$ such that $p_{1}, p_{2}$ and $p_{3}$ are rational, $\{x \in \partial N$ : $\left.\Re x=p_{1}\right) \subset F_{t}$ and $X_{s}$ is well defined. Theorem 2.6 implies easily that the set of points $X_{s}$ corresponding to such sets $N$ is dense in $\partial F_{t}$. For a fixed $t>0$, w.p.1, in every neighborhood of every such point $X_{s}$ there is a point of $B_{t}^{F}(-b, b)$. This completes the proof in the case $b-a \in(0, \pi)$, for a fixed $t>0$.

(D) Now let $b-a=\pi$ and assume without loss of generality that $b=-a=\pi / 2$. Fix some $x \in \mathbb{C}$ and let $N$ be the greatest closed square with center $x$, sides parallel to the axes, and such that its interior does not contain any points of $A_{t}$. It may be shown in a way completely analogous to the proof of Theorem 2.3 (i) that for a fixed $t>0$, w.p.1,

a) $N \cap A_{t}$ contains only one point $y=y(x)$ which is not a vertex of $N$ and

b) for some $b_{2} \in\{0, \pi / 2, \pi,-\pi / 2\}, \epsilon_{1}>0$, and all $a_{1} \leq b_{2}-\pi, b_{1} \geq b_{2}, b_{1}-a_{1}>$ $\pi, \epsilon_{2}>0$, one has $G\left(y, b_{2}-\pi, b_{2}, \epsilon_{1}\right) \subset N$ and $G\left(y, a_{1}, b_{1}, \epsilon_{2}\right) \cap A_{t} \neq \emptyset$.

Thus $y \in B_{t}\left(b_{2}-\pi, b_{2}\right)$; moreover, if $x \in F_{t}$ then $y \in B_{t}^{F}\left(b_{2}-\pi, b_{2}\right)$. For a fixed $t>0$, w.p.1 $y(x) \in B_{t}^{F}\left(b_{2}-\pi, b_{2}\right)$ simultaneously for all $x \in F_{t}$ with rational coordinates. It follows from Theorem 2.6 that the set of points $y=y(x)$ such that $x$ has rational coordinates, $x \in F_{t}$ and $y \in B_{t}^{F}(-\pi / 2, \pi / 2)$, is dense in $\partial F_{t}$.

(E) Let $b-a=0$, say $a=b=0$. Fix $t>0$ and denote $W_{s}=W_{s}^{q_{1}}=X\left(q_{1}+s\right)$. W.p.1, ${ }^{W} V_{q_{2}} \in{ }^{W} B_{q_{2}}^{F}(0,0)$ simultaneously for all pairs of rationals $q_{1}, q_{2} \geq 0$. Since the left endpoint of every interval of $\mathbb{R}_{+} \cap F_{t}$ is equal to ${ }^{W} V_{q_{2}}$ for some rational $q_{1}, q_{2}$, it follows that all such interval endpoints belong to $B_{t}^{F}(0,0)$. By the translation invariance, w.p.1 all left endpoints of intervals of $\left(x+\mathbb{R}_{+}\right) \cap F_{t}$ belong to $B_{t}^{F}(0,0)$, for all $x \in \mathbb{C}$ with rational coordinates. Theorem 2.6 implies easily that the set of such endpoints is dense in $\partial F_{t}$.

(F) Part (i) of Theorem 2.4 has been proved for $b-a \in[0, \pi]$, for a fixed $t>0$ and, therefore, it holds w.p.1 for all rational $t>0$ simultaneously.

Fix $a, b, b-a \in[0, \pi]$, and suppose that $B_{t}^{F}(a, b)$ is not dense in $\partial F_{t}$ for some $t>0$. Choose $x \in \partial F_{t}$ and $\epsilon>0$ such that $\left|x-X_{t}\right|>2 \epsilon$ and

$$
\left\{z \in B_{t}^{F}(a, b):|x-z|<\epsilon\right\}=\emptyset .
$$

Then, by continuity of $X$,

$$
\left\{z \in B_{s}^{F}(a, b):|x-z|<\epsilon\right\}=\emptyset
$$

for all $s \in\left(t, t+\epsilon_{1}\right)$ for some $\epsilon_{1}>0$, in particular for some rational $s_{1} \in\left(t, t+\epsilon_{1}\right)$. The last event has probability 0 , so w.p. $1 B_{t}^{F}(a, b)$ is dense in $\partial F_{t}$ for all $t>0$. This completes the proof of Theorem 2.4 (i).

ii) Theorem 2.4 (ii) follows from Corollary 2 (ii) of Evans (1985).

Proof of Theorem 2.5. i) Theorem 2.5 (i) follows from the results of Le Gall (1987). For $b-a \in[0, \pi]$, it is an immediate consequence of Theorem 2.4 (i).

ii) Part (ii) of Theorem 2.5 follows easily from the results of Burdzy (1985) and Shimura (1985). 


\section{Twist points.}

Proof of Theorem 2.6. i) Here is the outline of the proof.

(A) Each point of $x \in \partial F_{t}$ which is "nice" corresponds to a special set $J$.

(B) It suffices to show that every set $J$ corresponds to a negligible set of "nice" points.

(C) The assumption that $J$ corresponds to a nonnegligible set of "nice" points leads to a contradiction.

(D) McMillan's Theorem is used to show that almost all points of $\partial F_{t}$ are "twist points".

(A) Fix $t>0$. First, some more notation will be introduced. For a set $D \subset$ $\mathbb{C}, x \subset \mathbb{C}$ and $\epsilon>0$ let $h: \mathbb{R} \rightarrow[-\infty, \infty)$ be the smallest Lipschitz function with constant 3 such that

$$
\begin{gathered}
\{y \in \partial D:|\Re x-\Re y| \leq \epsilon,|\Im x-\Im y| \leq \epsilon\} \\
\subset\{y \in \mathbb{C}:|\Re x-\Re y| \leq \epsilon,|\Im x-\Im y| \leq \epsilon, \Re y \leq h(\Im y)\} .
\end{gathered}
$$

To see that such a function exists, notice that for a large $c$, the constant function $h \equiv c$ satisfies the above condition. Then $h$ may be defined as the infimum of the family of all Lipschitz functions with constant 3 satisfying the condition (notice that the Lipschitz constant is preserved under this operation). The function $h$ may be identically $-\infty$. With the above definition of $h$, let

$$
\begin{aligned}
J_{1}(x, \epsilon, D) & =\{y \in \mathbb{C}:|\Re x-\Re y|<\epsilon,|\Im x-\Im y|<\epsilon, \Re y>h(\Im y)\}, \\
J(x, \epsilon, c) & =e^{-i c} J_{1}\left(e^{i c} x, \epsilon, e^{i c} F_{t}\right), \quad c \in \mathbb{R} .
\end{aligned}
$$

Recall from Section 3(ii) the idea of the (partial) identification of the Euclidean and Martin boundaries of $F_{t}$. Let $f: D_{*} \rightarrow F_{t}$ be analytic, one-to-one and onto $\left(D_{*}=\{z \in \mathbb{C}: \Re z>0\}\right)$. Consider a point $x \in \partial D_{*}$ such that mf- $f^{\prime}(x)=a \in$ $\mathbb{C} \backslash\{0\}$. Choose $\epsilon>0$ so that $|\arg y-\arg a|<\pi / 12$ for $|a-y|<\epsilon$ and let $A=\left\{y \in D_{*}:\left|f^{\prime}(y)-a\right|<\epsilon\right\}$. The set $A$ is simply connected, by the maximum principle. It is a minimal fine neighborhood of $x$ in $D_{*}$, by the assumption that mf- $f^{\prime}(x)=a$. Thus there exists a Lipschitz function $h: \mathbb{R} \rightarrow \mathbb{R}$ with constant 1 such that $h(r)>0$ for $r \neq \Im x$,

$$
B \stackrel{\mathrm{df}}{=}\left\{y \in \mathbb{C}:|y-x|<\epsilon_{1}, \Re y>h(\Im y)\right\} \subset A
$$

for some $\epsilon_{1}>0$, and $B$ is a minimal fine neighborhood of $x$ in $D_{*}$ (see Section 3 (ii)). Observe that $\left|\arg f^{\prime}(y)-\arg a\right|<\pi / 12$ for $y \in \partial B, y \neq x$. It follows that for some neighborhood $U$ of $f(x), f(\partial B) \cap U$ is the graph of a Lipschitz function with constant 2 (in a suitable coordinate system). Easy geometry shows that $z=f(x)$ satisfies the following condition

(7.1) there exist $y \in \mathbb{C}, \epsilon_{2}>0, c \in \mathbb{R}$ and a neighborhood $U_{1}$ of $z$ such that

a) $|z-y| \leq \epsilon_{2} / 2$,

b) $\Re y, \Im y, \epsilon_{2}$ and $c$ are rational,

c) $f(B) \cap U_{1} \subset J\left(y, \epsilon_{2}, c\right) \cap U_{1}$ and

d) $J\left(y, \epsilon_{2}, c\right) \subset F_{t}$.

The condition (7.1c) implies that $J\left(y, \epsilon_{2}, c\right)$ is a minimal fine neighborhood of $f(x)$ in $F_{t}$ (see Section 3 (ii)). 
(B) Now it will be proved that the set $N$ of all points $x \in \partial D_{*}$ with mf- $f^{\prime}(x) \in$ $\mathbb{C} \backslash\{0\}$ has null harmonic measure in $D_{*}$. By the conformal invariance of the harmonic measure, it is enough to show that $f(N) \subset \partial F_{t}$ has null harmonic measure in $F_{t}$. The first part of the proof has shown that it is enough to prove that the set of points $z \in \partial F_{t}$ satisfying (7.1) has null harmonic measure in $F_{t}$. The family of sets $J\left(y, \epsilon_{2}, c\right)$ considered in (7.1) is countable, so the problem may be further reduced to showing that for fixed $y, \epsilon_{2}, c$, w.p. 1 the set of points $z \in \partial F_{t}$, for which $J\left(y, \epsilon_{2}, c\right)$ satisfies (7.1), has null harmonic measure in $F_{t}$.

Fix $y, \epsilon_{2}, c$ and let $J=J\left(y, \epsilon_{2}, c\right)$. By the rotation invariance of Brownian motion, it may be assumed without loss of generality that $c=0$. Let $K$ denote the set of all points $z \in \partial F_{t}$ such that $z$ and $J$ satisfy (7.1). Assume that the harmonic measure of $K$ in $F_{t}$ is strictly positive. It will be shown that this assumption leads to a contradiction.

(C) For each $z \in K, J$ is a minimal fine neighborhood of $z$ in $F_{t}$, so $K \subset \partial J$. The probabilistic interpretation of the minimal fine topology (Section 3 (iii) (C)) implies that for all $x \in F_{t}$ and $y \in K$,

$$
\tilde{P}_{y}^{x}\left(\sup \left\{s \geq 0: Y_{s} \in J^{c}\right\}<R\right)=1
$$

and

$$
\tilde{P}^{x}\left(\sup \left\{s<T_{K}: Y_{s} \in J^{c}\right\}<T_{K} \mid T_{K}=T_{\partial F_{t}}\right)=1 .
$$

The symbols with a "wave" refer to a Brownian motion $Y$ independent from $X$ which has been used to define $F_{t}, K$ and $J$. The hitting times are defined relative to $Y$. Observe that $\tilde{P}^{x}\left(T_{K}=T_{\partial F_{t}}\right)>0$, by assumption.

Let $g(y)=\tilde{P}^{y}\left(T_{K} \leq T_{J^{c}}\right)$. The function $g$ is nonnegative and harmonic in $J$ so it is either strictly positive in $J$ or identically 0 in $J$. Suppose that $g(y) \equiv 0$ in $J$. Then $g(y)=0$ for all $y \in \mathbb{C}$. The Markov property implies that for $x \in F_{t}$ and $u>0$,

$$
\tilde{P}^{x}\left(T_{K} \circ \theta_{u} \leq T_{J^{c}} \circ \theta_{u}\right)=\tilde{P}^{x}\left[\tilde{P}^{Y_{u}}\left(T_{K} \leq T_{J^{c}}\right)\right]=\tilde{E}^{x} g\left(Y_{u}\right)=0
$$

Since

$$
\left\{\sup \left\{s<T_{k}: Y_{s} \in J^{c}\right\}<T_{K}\right\} \subset \bigcup_{\text {rational } u}\left\{T_{K} \circ \theta_{u} \leq T_{J^{c}} \circ \theta_{u}\right\}
$$

it follows that

$$
\tilde{P}^{x}\left(\sup \left\{s<T_{K}: Y_{s} \in J^{c}\right\}<T_{K} \mid T_{K}=T_{\partial F_{t}}\right)=0,
$$

contrary to (7.2). This contradiction implies that $g(y)>0$ for $y \in J$.

Let $W(a)$ be the left endpoint of the maximal interval of $(\mathbb{R}+a i) \cap F_{t}$ which has a nonempty intersection with $J$, for $a \in\left(\Im y-\epsilon_{2}, \Im y+\epsilon_{2}\right) \stackrel{\text { df }}{=}\left(b_{1}, b_{2}\right)$. For a fixed $a$,w.p.1 $W(a) \in B_{t}^{F}(0,0)$ (see the proof of Theorem $2.4(\mathrm{i})$ ). Thus

$$
E^{0} \int_{b_{1}}^{b_{2}} \mathbf{1}_{\left\{W(a) \notin B_{t}^{F}(0,0)\right\}} d a=\int_{b_{1}}^{b_{2}} P^{0}\left(W(a) \notin B_{t}^{F}(0,0)\right) d a=0 .
$$

In other words, w.p.1 the set

$$
Z_{1} \stackrel{\text { df }}{=}\left\{x \in \mathbb{C}: \Re x=0, \Im x \in\left(b_{1}, b_{2}\right), W(\Im x) \notin B_{t}^{F}(0,0)\right\}
$$


has (1-dimensional) Lebesgue measure 0 . Let $Z_{2}$ be the projection of $Z_{1}$ onto the boundary of $J$ in the direction of the real axis. Since the boundary of $J$ is (locally) a graph of a Lipschitz function, it follows that the linear measure of $Z_{2} \subset \partial J$ is 0 . By a theorem of Dahlberg (1977), the harmonic measure of $Z_{2}$ in $J$ is null.

If $z \in K$ then $W(\Im z) \in \partial J$ and $W(\Im z) \notin B_{t}^{F}(0,0)$. Thus $K \subset Z_{2}$, the harmonic measure of $K$ in $J$ is 0 , and, consequently $g(y)=\tilde{P}^{y}\left(T_{K} \leq T_{J^{c}}\right)=0$ for $y \in J$. This is a contradiction which proves that the assumption that $K$ has a non-zero harmonic measure in $F_{t}$ must be false. According to previous remarks, this shows that the set of all points $x \in \partial D_{*}$ with $\mathrm{mf}-f^{\prime}(x) \in \mathbb{C} \backslash\{0\}$ has null harmonic measure in $D_{*}$.

(D) McMillan's Theorem (see Section 3(ii)) shows that almost all points $x \in \partial D_{*}$ are $f$-twist points. Hence, for a fixed $t>0$, w.p.1 all points of $\partial F_{t}$ are twist points, except a set of null harmonic measure.

To strengthen the result to floating $t>0$, use the same method as at the end of the proof of Theorem 2.4 (i).

ii) Part (ii) of Theorem 2.6 is an obvious corollary of part (i).

\section{REFERENCES}

1. Blumenthal, R,M. and Getoor, R.K., Markov Processes and Potential Theory, Academic Press, New York, 1968.

2. Burdzy, K., Brownian paths and cones, Ann. Probab. 13 (1985), 1006-1010.

3. , Brownian excursions from hyperplanes and smooth surfaces, Trans. Amer. Math. Soc. 295 (1986a), 35-57.

4. __ Brownian excursions and minimal thinness. Part III. Applications to the angular derivative problem, Math. Z. 192 (1986b), 89-107.

5.___ Brownian excursions and minimal thinness. I, Ann. Probab. 15 (1987a), 676-689.

6. __ Brownian excursions and minimal thinness. Part II. Applications to boundary behavior of the Green function (1987b), in Seminar on Stochastic Processes 1985. E. Cinlar, K. L. Chung, R. K. Getoor, eds., 35-62. Birkhäuser, Boston, (1987b)..

7. __ Multidimensional Brownian Excursions and Potential Theory, Longman, London, $1987 \mathrm{c}$.

8. _ Convergence of 2-dimensional h-processes, Probab. Math. Statist. 8 (1987d), 49-52.

9. __ Minimal fine derivatives and Brownian excursions, (1987e) (preprint)..

10. _ Cut points on Brownian paths, Ann. Probab. (1987f), (to appear)..

11. __ Harmonic measure on 3-dimensional Brownian paths, Math. Proc. Camb. Phil. Soc. (1988), (to appear).

12. Burdzy, K. and Williams, R.J., On Brownian excursions in Lipschitz domains. Part I. Local path properties, Trans. Amer. Math. Soc. 298 (1986), 289-306.

13. Cranston, M., Hsu, P. and March, P., Smoothness of the convex hull of planar Brownian motion, (1987) (preprint)..

14. Dahlberg, B.E.J., Estimates of harmonic measure, Arch. Rational Mech. Anal. 65 (1977), 275-288.

15. Doob, J.L., Classical Potential Theory and Its Probabilistic Counterpart, Springer-Verlag, New York, 1984.

16. El Bachir, M., L'enveloppe convexe du mouvement brownien, Th. 3-ème cycle. Université Toulouse III, (1983)..

17. Evans, S.N., On the Hausdorff dimension of Brownian cone points, Math. Proc. Camb. Phil. Soc. 98 (1985), 343-353.

18. Itô, K., Poisson point processes attached to Markov processes, Proc. Sixth Berkeley Symp. III (1972), 225-239, Univ. of California Press, Berkeley..

19. Itô, K. and McKean, H. P., Diffusion Processes and Their Sample Paths., Academic Press, New York, 1974, 2-nd ed..

20. Jackson, H.L., On the boundary behaviour of BLD functions and some applications, Bull. $\mathrm{Cl}$. Sci. Acad. R. Belgique 66 (1980), 223-239. 
21. Knight, F.B., Essentials of Brownian Motion and Diffusions, Amer. Math. Soc., providence, 1981, Math. Surveys, no. 18.

22. Le Gall, J.-F., Mouvement brownien plan, cones et processus stables, C. R. Acad. Sci. Paris 302 (1986), 641-643.

23. ㄴ Mouvement brownien, cones et processus stables, Probab. Th. Rel. Fields 76 (1987), 587-627.

24. Maisonneuve, B., Exit systems, Ann. Probab. 3 (1975), 399-411.

25. Mandelbrot, B.B., The Fractal Geometry of Nature, Freeman \& Co., New York, 1982.

26. McMillan, J.E., Boundary behaviour of a conformal mapping, Acta Math. 123 (1969), 43-68.

27. Mountford, T.S., On the asymptotic number of small components created by planar Brownian motion, (1987) (preprint)..

28. Naim, L., Sur le role de la forntiére de R.S. Martin dans la théorie du potential, Ann. Inst. Fourier (Grenoble) 7 (1957), 183-281.

29. Ohtsuka, M., Dirichlet Problem, Extremal Length and Prime Ends, Van Nostrand Reinhold, New York, 1967.

30. Pommerenke, Ch., Univalent Functions, Vandenhoeck and Ruprecht, Göttingen, 1975.

31. Shimura, M., A limit theorem for two-dimensional conditioned random walk, Nagoya Math. J. 95 (1984), 105-116.

32. __ Excursions in a cone for two-dimensional Brownian motion, J. Math. Kyoto Univ. 25 (1985), 433-443.

33. Varadhan, S.R.S. and Williams, R.J., Brownian motion in a wedge with oblique reflection, Comm. Pure Appl. Math. 38 (1985), 405-443.

34. Williams, D., Diffusions, Markov Processes and Martingales, Wiley, New York, 1979, vol. 1..

35. Williams, R.J., Local time and excursions of reflected Brownian motion in a wedge, Publ. RIMS Kyoto Univ. 23 (1987), 297-319.

Mathematics Department, Seattle, WA 98195 